\title{
Microcontroller-Based Two-Way Radio Interoperabilty System Public Safety Communications
}

\author{
Adil Akasha Mohammed ${ }^{1}$, Rashid Saeed ${ }^{2}$, Ala Eldin Awouda ${ }^{3}$ \\ 1,2,3 Sudan university of science and technology Electronics Department, Sudan
}

*Corresponding Author: Adil Akasha Mohammed, Sudan University of science and technology Electronics Department, Sudan

\begin{abstract}
This paper introduces an automatic controlled system for interoperability between different twoway radio networks that can cancel any third party may exist in interconnections systems. It depends upon the infrastructure and environmental condition of radio networks already exist and directly interest in voice communications by accessing audio through common featured radios accessory sockets. RF CALL signal along with the DC superimposed in audio are the controls of selecting networks connections and the status of the TX radio of the selected network respectively. The control signals are brought to control unit, microcontroller, to form the control center of the system. The first responder can connect directly with the agency concerned without need to be directed.
\end{abstract}

Keywords: interoperability; two-way radio; microcontroller

\section{INTRODUCTION}

The need of interoperability in communications is urgent .It affects every level of government as well as citizen trust in government. The problem is that when different first-responder organizations appear at an incident scene, their radios are incompatible, operate over different frequencies and use different techniques ${ }_{[1],[14]}$. The consequences, underscored are uncoordinated responses and a fragmented chain of command that can hinder the ability to save lives, property, and infrastructure. Communications interoperability is also recognized as an element of effective day-to-day public safety services. Members might include government agencies, private assistance groups, and enterprises-all of which might be using incompatible radio systems ${ }_{[11],[17]}$.

Public safety agencies use several methods such as swapping radios, mutual-aid channels, and gateways that bridge two or more radio systems. All of these provide some benefit, but they do not completely solve the limitations of radio communications ${ }_{[3]}$. The limitations include lack of standards, using devices other than radios are not able to communicate from outside the radio range, first responders from different networks need the ability to communicate with other networks in case of emergency, the radio spectrum is fragmented due to FCC lows, Commercial standards-based technologies is not available, radio equipment has a very long lifecycle, and funding is infrequent ${ }_{[9]}$.

\subsection{Public Safety Interoperability}

Developments are made to increase the urgency of communications interoperability in public safety, for the need for intelligent, responsive networks and the increase of attention paid to protecting facilities, critical infrastructure in the public and private sector, emergency response services, and weather-related disasters [22], [12].

They also apply to day-to-day public safety operations as well as emergency response. Examples include the Olympic Games and other major sporting events, political and diplomatic events, and conventions. Similarly, agencies increasingly expect to collaborate during crimes that span them, such as car chases, gang crimes, and Amber alerts [6].

\subsection{Interoperability Approach}

There are some old solutions for interoperability such as swapping radio, channel sharing, relying dispatchers, radio gateways, etc.. [7],[15]. Also there are advanced solutions such as coalition peering 
domain, software defined radio (SDR), wireless gateways and directional antenna, project 25 (P25), cellular and internet, etc.. [14] [23].. [32][22]],[15],[16]],[33],[34]],[43],[45]],[46].

\section{The CaSe for Controller Design}

The design represents the third party console with microcontroller and control signals to assign the connections.

\section{A. System Architecture}

The overall design of the system contains three two-way radio networks with different bands, HF, VHF and UHF which connect with each other by extracting audio from network and diverted to another network for transmission by using a programmed control unit driven by two CALL signals in order to select one of the other two networks. Fig.2.

\section{B. Connections algorithm}

In table [1] the CALL signals are only two for they are three networks i.e the CALL signals is proportional to the number of networks in the system.ACK in a control signal generated from the audio signal. TX is the transmitter status.

Table1. Connections Algorithm

\begin{tabular}{|c|c|c|c|c|c|c|c|c|c|c|c|c|}
\hline \multicolumn{4}{|c|}{$H F$} & \multicolumn{4}{|c|}{ VHF } & \multicolumn{4}{|c|}{ UHF } & \multirow{3}{*}{$\begin{array}{c}\text { CONNECTIONS } \\
\text { STATUS }\end{array}$} \\
\hline \multicolumn{2}{|c|}{ CALL } & \multirow[t]{2}{*}{ ACK } & \multirow[t]{2}{*}{ TX } & \multicolumn{2}{|c|}{ CALL } & \multirow[t]{2}{*}{ ACK } & \multirow[t]{2}{*}{$T X$} & \multicolumn{2}{|c|}{ CALL } & \multirow[t]{2}{*}{ ACK } & \multirow[t]{2}{*}{ TX } & \\
\hline low & high & & & low & high & & & low & high & & & \\
\hline 1 & 0 & 1 & 0 & - & - & - & 1 & - & - & - & - & HF-to-VHF \\
\hline 0 & 1 & 1 & 0 & - & - & - & - & - & - & - & 1 & HF-to-UHF \\
\hline- & $\cdot$ & - & 1 & 1 & 0 & 1 & 0 & - & - & - & - & VHF-to-HF \\
\hline- & $\cdot$ & - & - & 0 & 1 & 1 & 0 & - & - & - & 1 & VHF-to-UHF \\
\hline- & - & - & 1 & - & - & - & - & 1 & 0 & 1 & 0 & UHF-to-HF \\
\hline$\cdot$ & - & $\cdot$ & - & $\cdot$ & $\cdot$ & - & 1 & 0 & 1 & 1 & 0 & UHF-to-VHF \\
\hline
\end{tabular}

\section{System description}

This interoperability system is designed to link different two-way radio networks with different bands such as HF, VHF, UHF, with each other as in fig. 2.

A frequency called CALL is used to control the switching between bands, for example, for VHF band the CALL frequency is chosen in the same VHF range. The call frequency received at a center room filtered, amplified and rectified. Fig. 3 ,the resulted DC through a single transistor switching circuit can ground PIN 1 on PORT A of the microcontroller which interns connects the audio of RX RADIO[VHF] AGENT 1 at its PORT B PIN 1 to the MIC IN of TX RADIO[UHF] AGENT 2 at its PORT C PIN 1. The microcontroller send confirmation tone through PIN 3 PORT B by grounding PIN 2 PORT B to put AGENCY 1 RADIO in the TX mode, then the microcontroller waits for AUDIO AKN at PORT B PIN 4to be LOW and ground PIN 2 PORT C put AGENCY 2 RADIO in the TX mode and the audio from RX AGENCY 1 is already connected to the MIC IN of TX AGENCY 2 .The microcontroller terminates the connection after waiting for a given time when AUDIO AKN PIN of RX AGENCY 1 goes HIGH.

\section{System Component}

- LNA[low noise amplifier]

A single wide band LNA is enough for any number of radio networks according to 


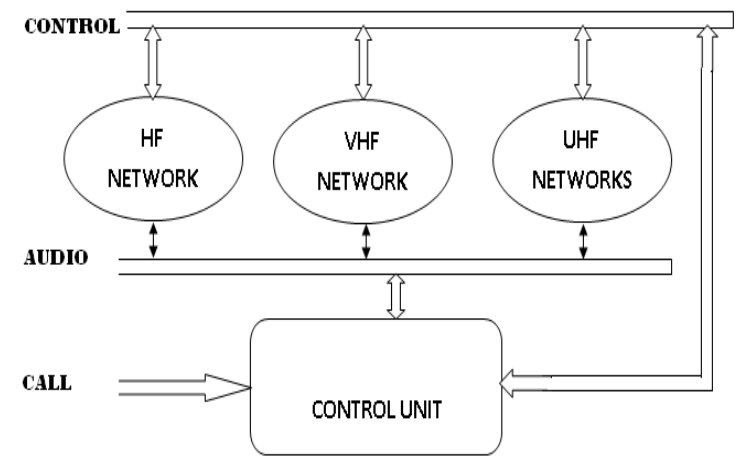

Fig2. System architecture

Telecommunications components products market. Another signal amplifier is also necessary for the signal to reach the desired level of about $5 \mathrm{~V}$.

\section{- Filter Unit}

This contains an RF filters. The number of RF filter circuits are related to the number of CALL frequencies depending upon the number of the radio networks sharing the system. Any CALL frequency filtered by a well calculated band pass filter using suitable (FILTER CAD) software. As in Fig.3. The filtered CALL frequencies is then rectifiered to obtain the desired logic level, fig. 4.

\section{- MCU ATMEGA16}

The logic values obtained are used, through suitable software, to connect the networks through their radio accessory or MIC pins, mainly PTT,AUDIO IN and AUDIO OUT ,depending on the logic value at $\mathrm{PIN}(\mathrm{X})$ on $\mathrm{PORT}(\mathrm{Y})$. When the logic level is correct a confirmation tone is fed back to begin data or voice transmission. Fig.5.

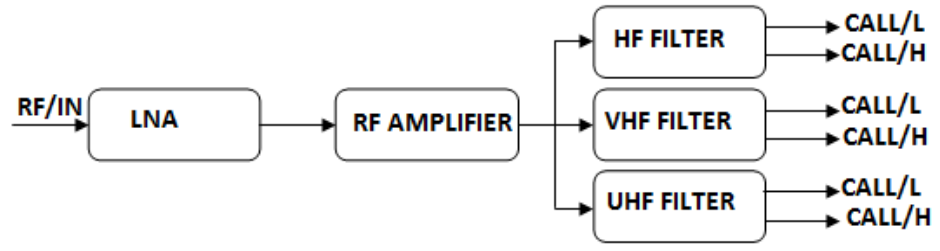

Fig3. Bands Filter Classifications

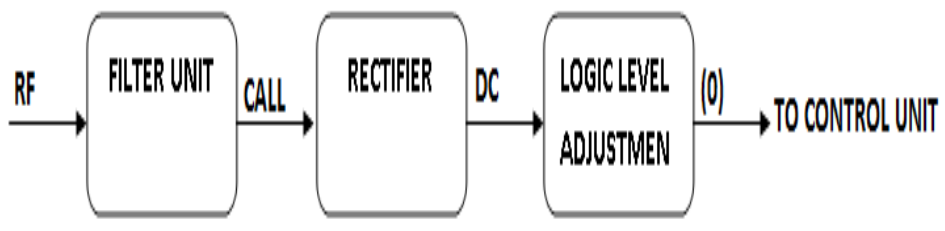

Fig4. Control Signals Generation

\section{E. Hardware Flowchart}

The system hardware is explained in Fig. 5. Where CH denotes HF network, and CV for VHF network while CU denotes UHF network.

\section{F. System Flow Chart}

Reading port A 0 5 are as follows:

- Pin 0,1 are used to assign CALL from HF network ,the arrival of CALL at pin 0 means the connection is with the VHF network, while pin 1 is for UHF network. Port B pin 0 indicates the arrival of audio signal which controls the status of port $\mathrm{C}$ pin 0 and pin 1

- Pin 2,3 are used to assign CALL from VHF network ,the arrival of CALL at pin 2 means the connection is with the HF network, while pin 3 is for UHF network. Port B pin 1indicates the arrival of audio signal which controls the status of port $\mathrm{C}$ pin 1 and pin 2 
- Pin 4,5 are used to assign CALL from UHF network, the arrival of CALL at pin 4 means the connection is with the VHF network, while pin 5 is for HF network. Port B indicates the arrival of audio signal which controls the status of port $\mathrm{C}$ pin 0 and pin 2.Fig. 6.

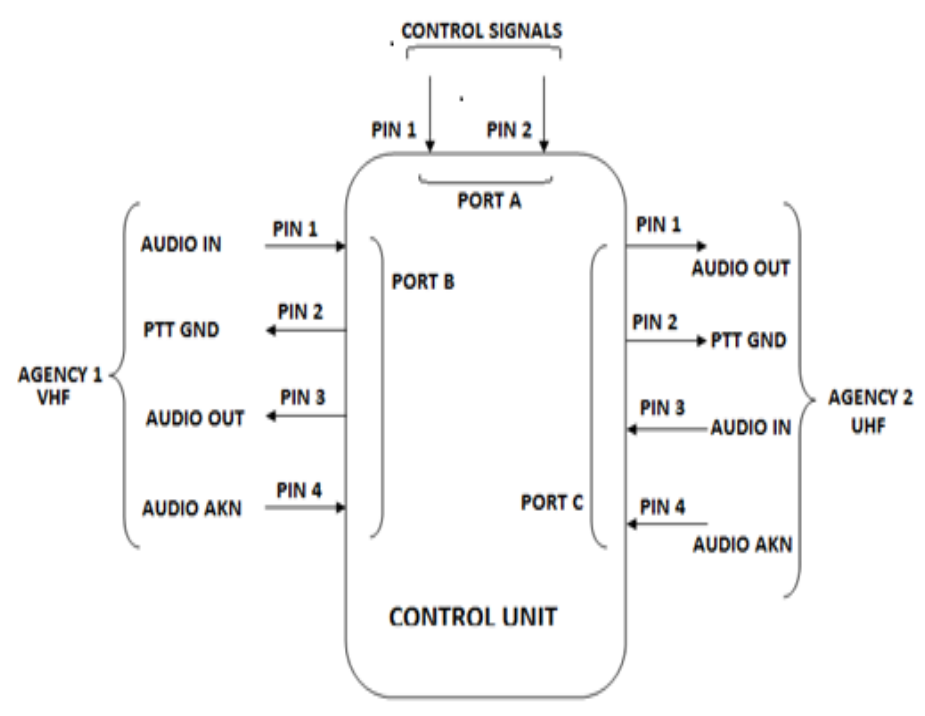

Fig5. Control Unit

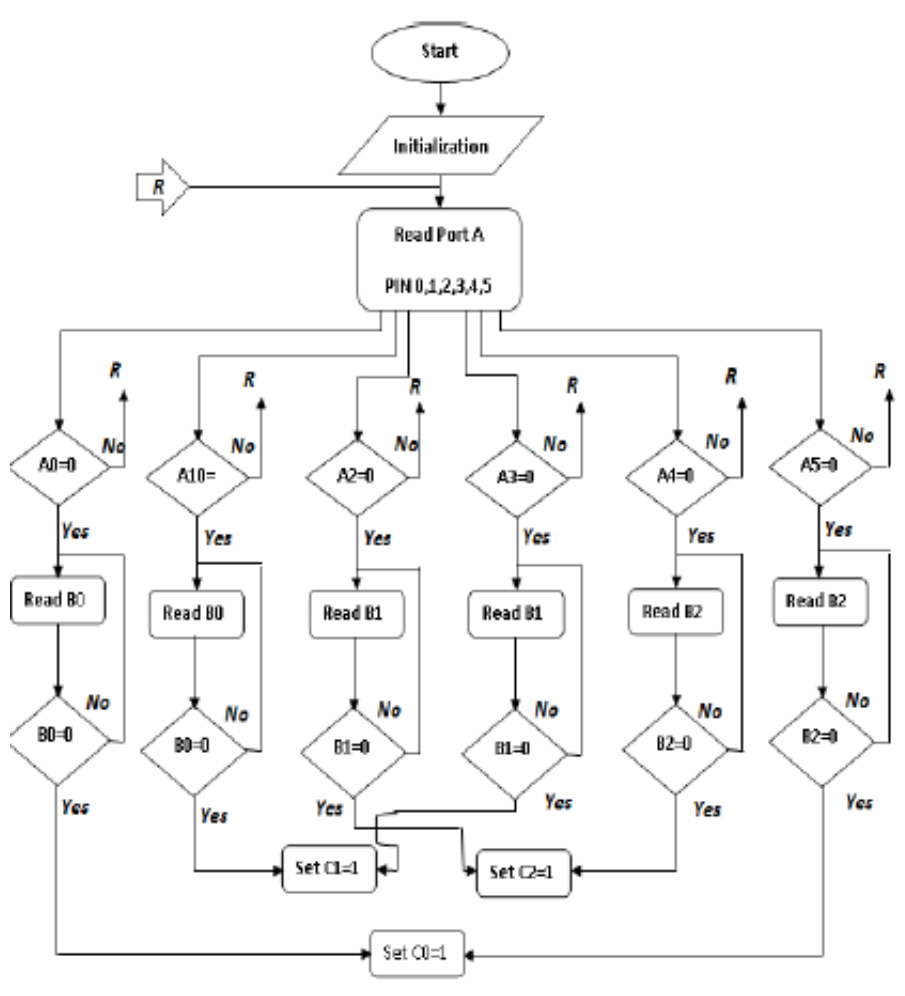

Fig6. System Flow Chart

\section{Simulation RESUlts}

The simple rectifier circuit converts the relatively high frequency into smooth DC that when applied to [U1] analogue switch [ DG418] which in turns grounding the controller selected PIN. Fig. 7.

Another control output control signal is generated so as to put the TX radio of the selected other side network in the TX mode using the analog switch [U1] [DG418] .Fig. 8.

Another control output control signal is generated so as to put the TX radio of the selected other side network in the TX mode using the analog switch [U1] [DG418] .Fig. 8. 
Fig. 9 shows the main body of the system CALLs and ACKs are represented by ON/OFF switches ,where the selected network is indicated by LEDs.

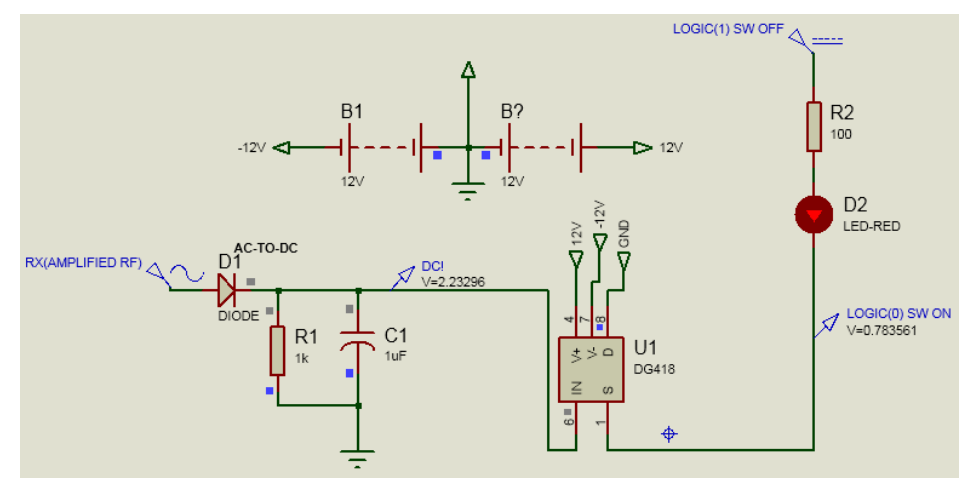

Fig7. The generation of the control signal

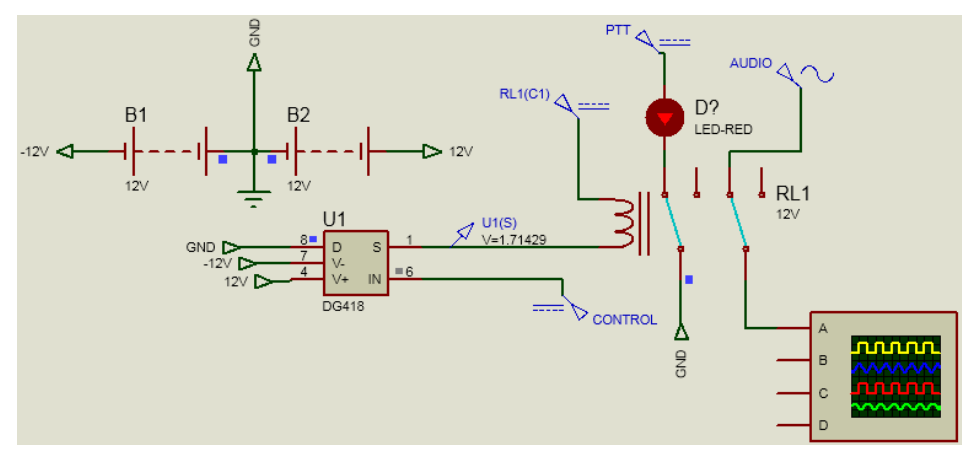

Fig8. Putting radio in TX mode and transmits data

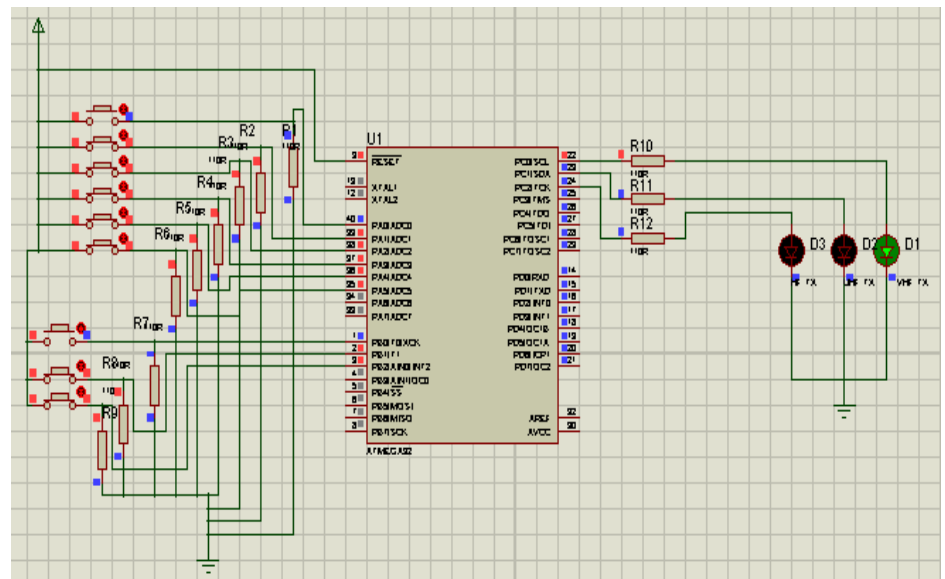

Fig9. Controller IN/OUT Signals

\section{CONCLUSiON}

The system do not needs new hardware architecture so the system is said to be compatible, also the system cancels the lack of time spends by consoler. Under good communications media networks, first responder can directly connected to the desired agency network through his own network. In some times it is difficult to establish connection because of high noise level that affect the logic values by error or undetermined status, retrying may not be helpful because it can be network matter or whether conditions.

\section{REFERENCES}

[1] Venkataraman, H., Sinanovic, S., \& Haas, H. (2008). Cluster-based design for two-hop cellular networks. International Journal for Communications, Networks and Systems (IJCNS), 1(4), 370-385

[2] Ikki, S. S., \& Ahmed, M. H. (2010). Performance analysis of best-path selection scheme for multi-hop amplify-and-forward relaying. European Transaction on Telecommunications,21(7), 603-610.

[3] Ann, S., \& Kim, H. S. (2010). Relay association method for optimal path in IEEE 802.16j mobile multihop relay networks. European Transaction on Telecommunications,21(6), 624-631. 
[4] Huang, J. H., Wang, L. C., Chang, C. J., \& Su, W. S. (2010). Design of optimum relay location in two-hop cellular systems.ACM/Springer Wireless Networks,16(8), 2179-2189.

[5] Xie, R., Richard Yu, F., \& Ji, H., (2011). Joint power allocation and beam forming with users selec-tion for CR networks via discrete stochastic optimization.ACM Wireless Networks. doi:10.1007/s11276-0120413-8.

[6] Bian, K., \& Park, J. M. (2006). MAC-layer misbehaviours in multi-hop cognitive radio networks. In International conference on science, technology, and entrepreneurship (UKC), Korea, August 2006.

[7] Shin, J., Lee, D. K., \& Cho, H. S. (2009). Is a multi-hop relay scheme gainful in an IEEE 802.22-based cognitive radio system. IEICE Transactions on Communications,92, 3528-3532.

[8] Zhuo Sun and Wenbo Wang "Investigation of Cooperation Technologies in Heterogeneous Wireless Networks", Journal of Computer Systems, Networks, and Communications, Volume (2010), Article ID 413987.

[9] Ermolov V. et al. "Significance of Nanotechnology for future wireless devices and Communications", The 18th Annual IEEE International Symposium on PIMRC'07. International Journal of Next-Generation Networks (IJNGN) Vol.4, No.3,September 2012 .

[10] R.K.Jain, Risal Singh, "Role of Nanotechnology in future wireless and communication systems", National seminar proceeding, Academy of Business \& Engineering Science Ghaziabad, pp-19-28, 16-17th January 2009.

[11] Peter Mell and Timothy Grance, "The NIST Definitionof Cloud Computing" US National Institute of Standards and Technology Special Publication 800-145, September 2011.

[12] H. Zhang, Y. Zheng, M. A. Khojastepour, and S. Rangarajan, .Cross-Layer Optimization for Streaming Scalable Video over Fading Wireless Networks,. in IEEE JSAC, vol. 28, no. 3, April 2010

[13] Hulse, Jonathan, and Bowen Tritter. "Transit Agency Implementation of, or Migration to Digital Trunked Radio." 2013 Rail Conference .2013.

[14] Atkinson, Randall, et al. "A proposal for coalition networking in dynamic operational environments. "Military Communications Conference, 2006. MILCOM 2006. IEEE. IEEE, 2006.

[15] De Sanctis, Mauro, et al."Interoperability solutions between smartphones and wireless sensor networks. "2012 IEEE first AESS European conference on satellite telecommunications (ESTEL) .2012.

[16] Dorfinger, P., et al."Self-aligning wireless communication for first responder organizations in interopera ble emergency scenarios." Proc. of the 2014 International Conference on Wireless Networks (ICWN 2014) 2014.

[17] Mousa, Anwar M. "Prospective of fifth generation mobile communications."International Journal of NextGeneration Networks (IJNGN) 4.3 (2012): 1-30.

[18] Venkataraman, Hrishikesh,Dipesh Gandhi, and Vikrant Tomar. "Multi-hop Multi-band Intelligent RelayBased Architecture for LTE-Advanced Multi-hop Wireless Cellular Networks." Wireless personal communications 75.1 (2014):131-153.

[19] Gupta, Piyush, and Priyadarshan Patil. "4G-a new era in wireless telecommunication." Magister Program in S/W Engineering, Malardalen University (2009).

[20] $\mathrm{Xu}, \mathrm{Xu}$, et al. "Maximizing network lifetime via 3G gateway assignment in dual-radio sensor networks." Local Computer Networks (LCN),2012 IEEE 37th Conference on .IEEE, 2012.

[21] Ouyang, Ye, and M. Hosein Fallah. "A performance analysis for UMTS packet switched network based on multivariate KPIs." Wireless Telecommunications Symposium (WTS), 2010 . IEEE,2010.

[22] Raluca, M-E., M-E. Razvan,and Andreas Terzis. "Gateway design for data gathering sensor networks." Sensor,Mesh and Ad Hoc Communications and Networks, 2008. SECON'08.5th Annual IEEE Communications Society Conference on . IEEE, 2008.

[23] Kamruzzaman, S. M. "CR-MAC: A multichannel MAC protocol for cognitive radio ad hoc networks." arXiv preprint arXiv:1009.4521 (2010).

[24] Garhwal, Anita, and Partha Pratim Bhattacharya. "A survey on dynamic spectrum access techniques for cognitive radio." arXiv preprint arXiv:1201.1964 (2012).

[25] Chowdhury, Kaushik R., Marco Di Felice, and Ian F. Akyildiz."TP-CRAHN: A transport protocol for cognitive radio ad-hoc networks." INFOCOM 2009, IEEE . IEEE, 2009.

[26] Subhedar, Mansi, and Gajanan Birajdar. "Spectrum sensing techniques in cognitive radio networks: a survey." International Journal of Next- Generation Networks 3.2 (2011): 37-51.

[27] Chen, K-C., et al. "Cognitive radio network architecture: part I--general structure." Proceedings of the $2^{\text {nd }}$ international conference on Ubiquitous information management and communication. ACM, 2008. 
[28] Kim, Hyoil, and Kang G. Shin."In-band spectrum sensing in cognitive radio networks:energy detection or feature detection?." Proceedings of the 14th ACM international conference on Mobile computing and networking.ACM, 2008.

[29] Xu, Dan, Eric Jung, and Xin Liu. "Optimal bandwidth selection in multi-channel cognitive radio networks: how much is too much?." New Frontiers in Dynamic Spectrum Access Networks, 2008.DySPAN 2008. 3rd IEEE Symposium on . IEEE, 2008.

[30] Çamurli, Mustafa, and Didem Gözüpek. "Channel Switching Cost-Aware Resource Allocation for Multihop Cognitive Radio Networks with a Single Transceiver." Ad Hoc Networks . Springer International Publishing, 2014.158-168

[31] Peha, Jon M. "Sharing spectrum through spectrum policy reform and cognitive radio." Proceedings of the IEEE 97.4 (2009): 708-719.

[32] Net-Hobby, Patrick L., and David E. Petty. "Emergency radio communications system incorporating integral public safety radio bridging capability." U.S. Patent No. 8,934,934. 13 Jan. 2015.

[33] Unger, Timo Marcus. "Multi-antenna relay station with two-way channel." U.S. Patent No. 8,644,208. 4 Feb. 2014.

[34] Makhlouf, Isam R., Ethan Y. Chen, and Xiaowei Jin. "Methods and apparatus for detecting and mitigating radio interference among user equipment and base stations of geographically co-located and spectrally distinct wireless systems." U.S. Patent No. 8,655,304. 18 Feb. 2014.

[35] Johnsson, Kerstin, Shilpa Talwar, and Nageen Himayat. "Dynamic interference mitigation for cellular networks." U.S. Patent No. 8,700,085. 15 Apr. 2014

[36] Dabbs III, James M., and Brian Claise. "Method and apparatus for efficient and deterministic group alerting." U.S. Patent No. 9,014,659. 21 Apr. 2015.

[37] Brownrigg, Edwin B. "Citizens' emergency network system and method." U.S. Patent No. 8,630,301. 14 Jan. 2014.

[38] Shaffer, Shmuel, et al. "Interoperability and Communications System Dynamic Media Proxy Based on Capability Negotiation." U.S. Patent Application 14/523,299.

[39] Klein, David E., et al. "Enhanced public safety communication system." U.S. Patent No. 8,738,061. 27 May 2014.

[40] Nusev, Zoran, and Aleksandar Risteski. "Design Issues of Interoperable Communication System for Public Safety-Planning for the Unplanned." Computational Intelligence, Communication Systems and Networks (CICSyN), 2014 Sixth International Conference on. IEEE, 2014.

[41] Benmohamed, Lotfi, et al. "Communications Architecture for National Security and Public Safety: Multijurisdictional, Multitechnology Network of Interoperable Networks." Military Communications Conference (MILCOM), 2014 IEEE. IEEE, 2014.

[42] Hobby, Patrick L., and David E. Petty. "Emergency Radio Communications System Incorporating Integral Public Safety Radio Bridging Capability." U.S. Patent No. 20,150,147,996. 28 May 2015.

[43] Brand, Stephen, John Jurrius, and David Brand. "Multi-channel multi-access voice over ip intercommunication systems and methods." U.S. Patent Application 14/511,921.

[44] Contreras, Nereydo T., et al. "Multi-band subscriber antenna for portable two-way radios." U.S. Patent No. 8,884,838. 11 Nov. 2014.

[45] Economy, George R., and Daniel J. McDonald. "Method and apparatus for selecting one of a plurality of networks for an application service based upon performance metrics for the application service." U.S. Patent No. 8,989,740. 24 Mar. 2015.

[46] Trucco, P., et al. "The rise of regional programmes on critical infrastructure resilience: identification and assessment of current good practices." Disaster Management and Human Health Risk IV: Reducing Risk, Improving Outcomes 150 (2015): 233.

[47] Newkirk, Dennis R., et al. "Method and apparatus for providing application service between a first protocol and a second protocol." U.S. Patent No. 8,861,482. 14 Oct. 2014.

[48] Melvin, Darnelle Omar. "Managing Metadata Interoperability within Audio Preservation Framework: Integrating the Metadata Encoding \& Transmission Standard (METS) and Multichannel Source Material into Digital Library Audio Collections." Library Philosophy and Practice (2014): 0_1.

Citation: Adil Akasha Mohammed et al. (2018). Microcontroller-Based Two-Way Radio Interoperabilty System Public Safety Communications, International Journal of Innovative Research in Electronics and Communications (IJIREC), 5(1), pp.1-7, DOI: http:// dx.doi.org/10.20431/2349-4050.0501001

Copyright: (C) 2018 Adil Akasha Mohammed. This is an open-access article distributed under the terms of the Creative Commons Attribution License, which permits unrestricted use, distribution, and reproduction in any medium, provided the original author and source are credited 